\title{
Abducens nerve palsy on a patient with sphenoid fungal rhinosinusitis and cavernous sinus meningioma - Case report
}

\author{
Andreea Marza', Claudiu Manea ${ }^{1,2,3}$ \\ ${ }^{1}$ ENT\&HNS Department, "Sfanta Maria" Hospital, Bucharest, Romania \\ ${ }^{2}$ CESITO Center, "Sfanta Maria" Hospital, Bucharest, Romania \\ 3"Carol Davila" University of Medicine and Pharmacy, Bucharest, Romania
}

\begin{abstract}
We report a case of a 47-year-old patient recently diagnosed with left abducens nerve palsy, who was admitted in our clinic with diplopia. The cranio-facial CT scan revealed left sphenoid fungal rhinosinusitis and the patient underwent endoscopic surgery with complete removal of the fungal material. The immediate postoperative evolution of the patient was favourable, with partial improvement of diplopia. The first month follow-up visit revealed the recurrence of the diplopia, so the patient performed an MRI scan of the brain that showed a cavernous sinus meningioma. The patient was referred to a neurosurgeon who choose a "wait-and-see" strategy instead of surgery.

The paper presents the limits of computed tomography versus magnetic resonance imaging in the radiologic diagnosis of intracranial tumors.
\end{abstract}

KEYWORDS: abducens nerve palsy, sphenoid rhinosinusitis, cavernous sinus meningioma.

\section{INTRODUCTION}

Abducens nerve palsy is the most common cause of acquired extraocular muscle paralysis. The abducens nerve has a high vulnerability to direct and indirect injuries due to its long intracranial course alongside neural, vascular, ligamentous and bony structures $^{1-4}$. There are many ethiologies that can cause the abducens nerve palsy like trauma, tumor, infection, intracranial aneurysm and subarachnoid hemorrhage ${ }^{4}$, increased intracranial pressure, demyelinating disease, etc. The sixth nerve palsy is commonly caused by tumors that are located close to the nerve, such as tumors in the cavernous sinus. The tumor may compress the nerve directly, or the peritumoral edema may compress the nerve indirectly ${ }^{5,6}$. Horizontal gaze diplopia may occur in a sphenoid pathology due to the close proximity of the abducens nerve to the sphenoid sinus.

We present a case of abducens nerve palsy associated with sphenoid fungal rhinosinusitis and cavernous sinus meningioma, emphasising on the diagnostic and therapeutic management of this clinical case.

\section{CASE REPORT}

A 47-year-old patient was admitted to our clinic for horizontal diplopia with a 2-week evolution.

The patient was initially referred to the county hospital due to sudden onset of double vision. The clinical and paraclinical assessment of the patient excluded all the acute causes of diplopia. After the neurological examination, the patient was diagnosed with left abducens palsy. The patient performed a native computed tomography scan of the brain that revelead opacification of the left sphenoid sinus, without any signs of osteolysis. The ENT examination established the diagnosis of chronic rhinosinusitis and the patient received the recommendation of surgical sinus drainage. During the hospitalized days in the county hospital, the patient underwent neurotrophic vitamin therapy without any noticeable improvement in diplopia.

At the time of presentation in our department, the ENT clinical examination revealed a limitation of the left ocular globe excursions at the left lateral view. The nasal endoscopy identified mucopurulent discharge from the left sphenoethmoidal recess. The preopera-

Corresponding author: Claudiu Manea, MD, PhD, Associated Professor, ENT\&HNS Department, CESITO Center, "Sfanta Maria" Hospital, 37-39 Ion Mihalache Blvd., District 1, Bucharest, Romania 

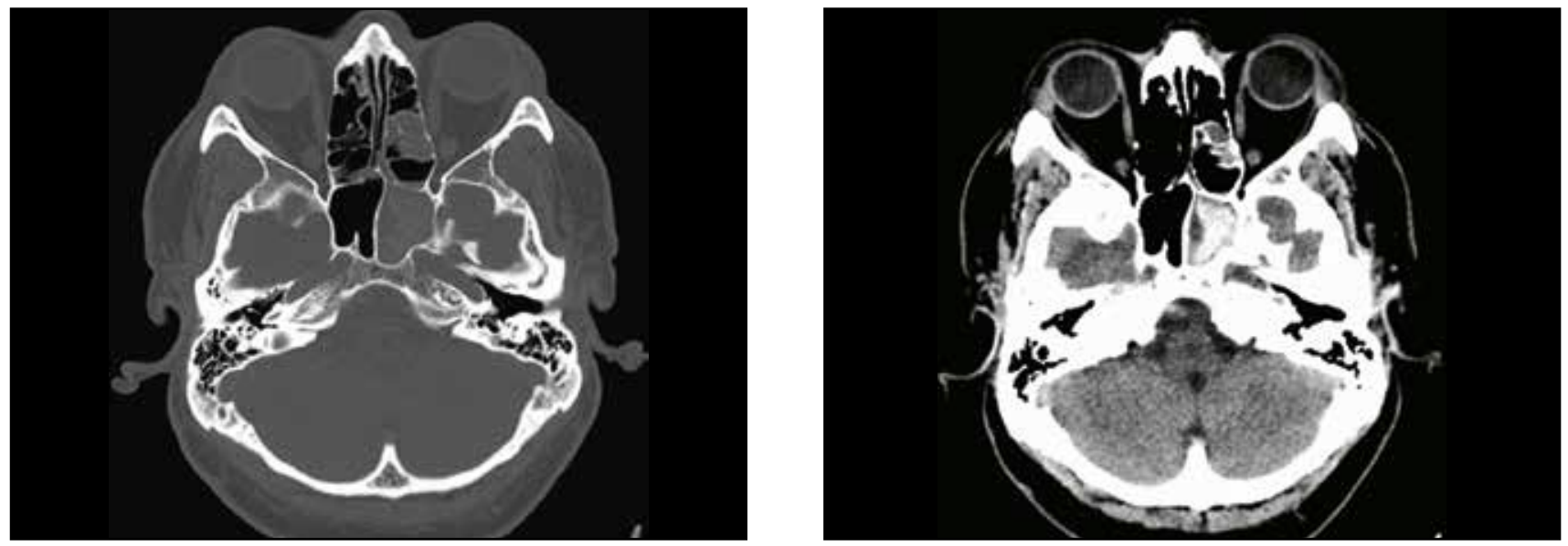

Figure 1 Preoperative cranio-facial CT scan, axial sections, showing opacification of the left sphenoid sinus with metallic densities.

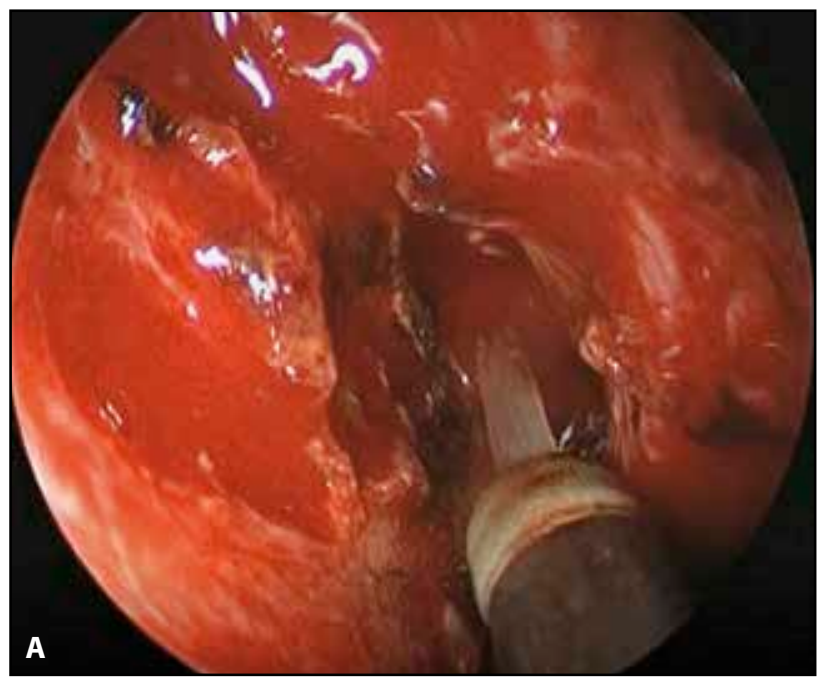

Figure 2 A) Eosinophilic mucin aspirated from the left sphenoid sinus after sphenoidotomy; B) Fungal material inside the sphenoid sinus; C) Left sphenoid sinus after removal of the fungal content, with granulomatous edema of the sinus mucosa.

tive CT scan images showed opacification of the left sphenoid sinus, without any signs of osteolysis, with areas of metallic densities intrasinusally, images that may indicate a fungal etiology (Figure 1).

The patient underwent transnasal endoscopic surgery for drainage of the left sphenoid sinus. A left transostial sphenoidotomy was performed with complete removal of sinus contents (eosinophilic mucin and abundant, brown-colored amorphous material, evocative for fungal material - Figure 2). At this point during the surgery, a sample was taken for histopathological and mycological examination. Lavage with physiological serum of the intrasinusal cavity and intranasal packing were performed. The medications received by the patient in the perioperative period included high doses of steroids and antibiotics.

Immediate postoperative evolution was favourable,
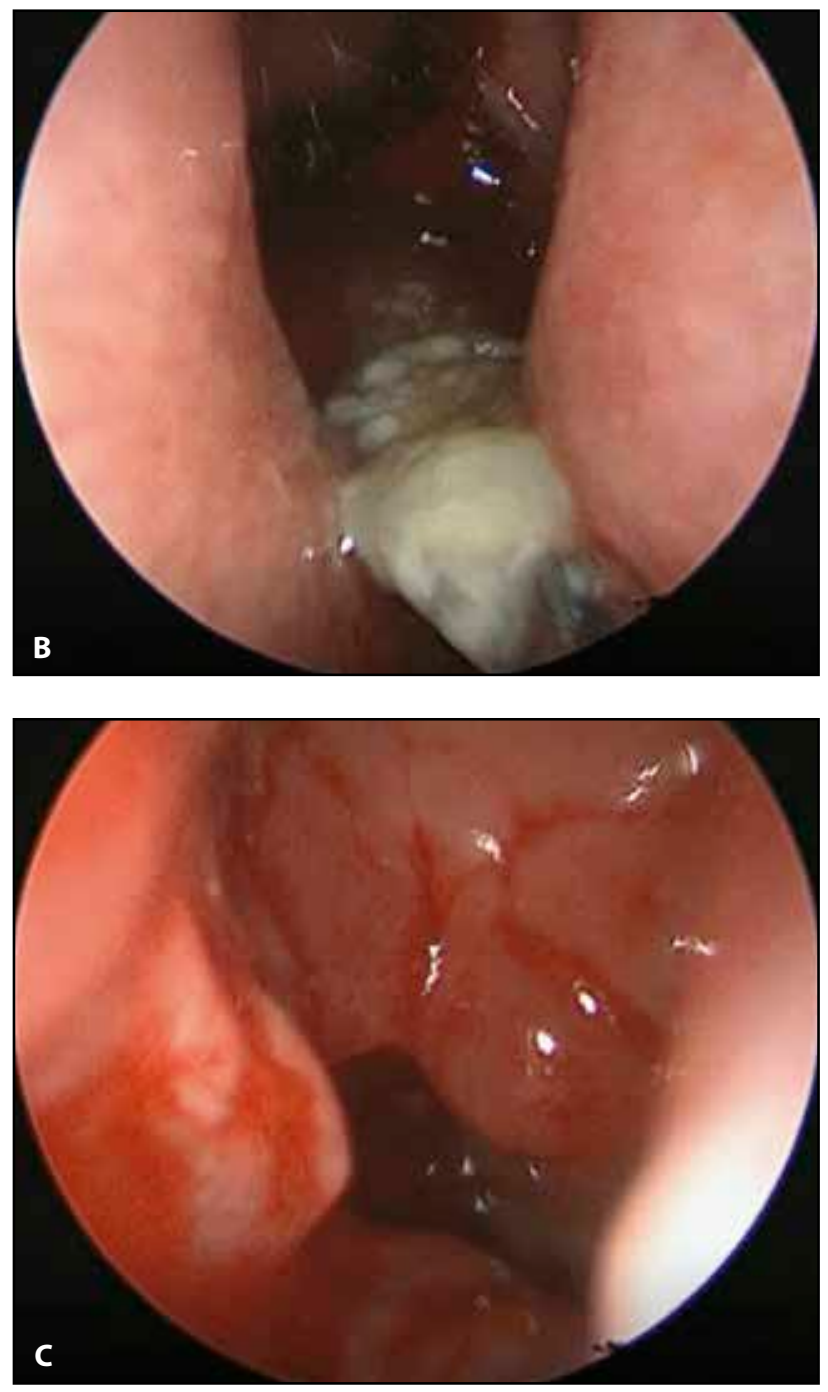

the patient being able to partially achieve abduction of the left eyeball, maintaining a degree of abduction limitation less than $60 \%$ comparing with the initial presentation. During the early postoperative period, subjectively, the patient reported improvement in diplopia. 

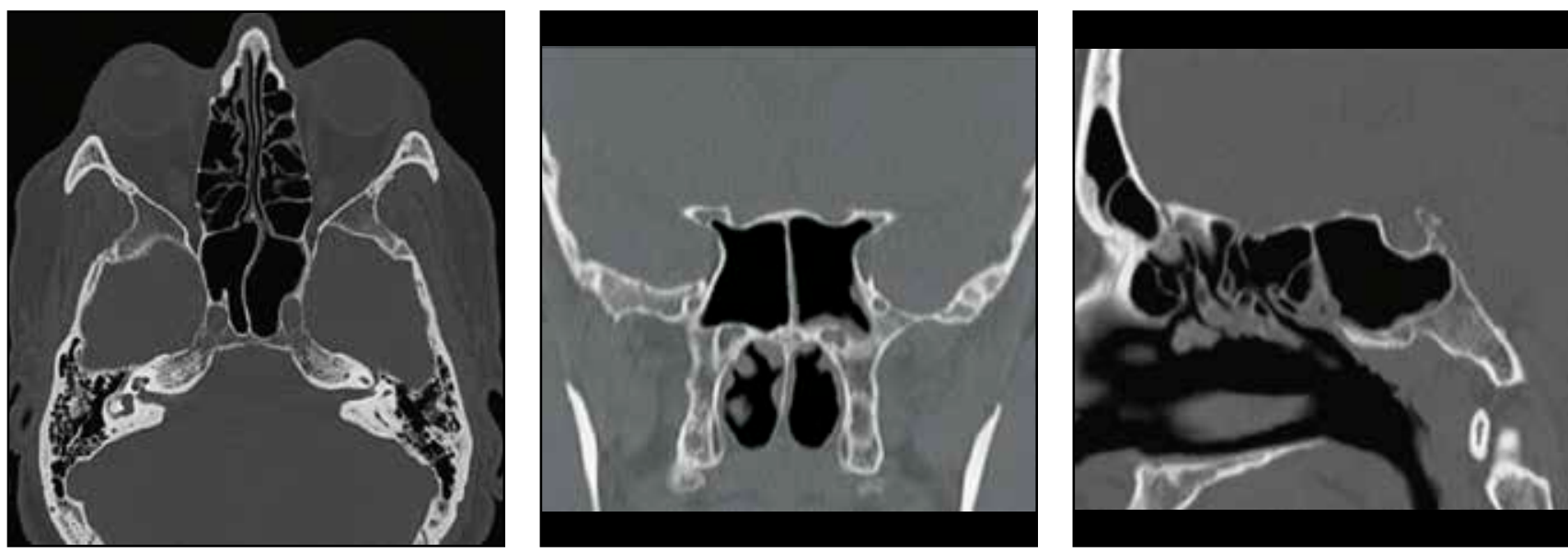

Figure 3 The postoperative cranio-facial CT scan (axial, coronal and sagital section) shows a normal pneumatization of the left sphenoid sinus, witout signs of infammation or osteolisis.

At the first month follow-up visit, the patient reported diplopia with progressively aggravating evolution, so we recommended a postoperative CT scan and a neurologic reexamination.

The postoperative CT scan showed a normal pneumatization of the left sphenoid sinus, without any signs of sinus infection (Figure 3).

The neurologist ordered an MRI scan of the brain with contrast agent (Gadolinium), which revealed a small mass involving the cavernous sinus on the left side and tentorium. The radiological aspect suggested a meningioma (Figure 4), so the patient was referred to a neurosurgery department. The neurosurgeon confirmed the abducens nerve palsy and the cavernous sinus meningioma. Because of the dimensions of the tumor and the involvement of the cavernous sinus, the neurosurgeon recommended a "wait-and-see" strategy, with a periodic MRI scan imaging evaluation instead of a surgical treatment at that time.

\section{DISCUSSIONS}

Meningiomas are tumors originating from meningothelial cells of the arachnoida. Most meningiomas are benign tumors, growing at a slow rate, corresponding to grade I of WHO histopathologic classification ${ }^{7}$. 5-7\% of all cases are atypical meningiomas, corresponding to grade II of WHO histopathologic classification, whereas only 1-3\% of all cases are reported to be malignant meningiomas, corresponding to grade III of WHO histopathologic classification ${ }^{8}$.

Due to the potential involvement with cranial nerves II-VI and the internal carotid artery, the most challenging lesions for the skull base surgeon are the meningiomas of the cavernous sinus. They arise from the dura of the cavernous sinus itself in most cases, but also from the dura of the sphenoid ridge, clinoid processes, petroclival region, or anterior skull base that
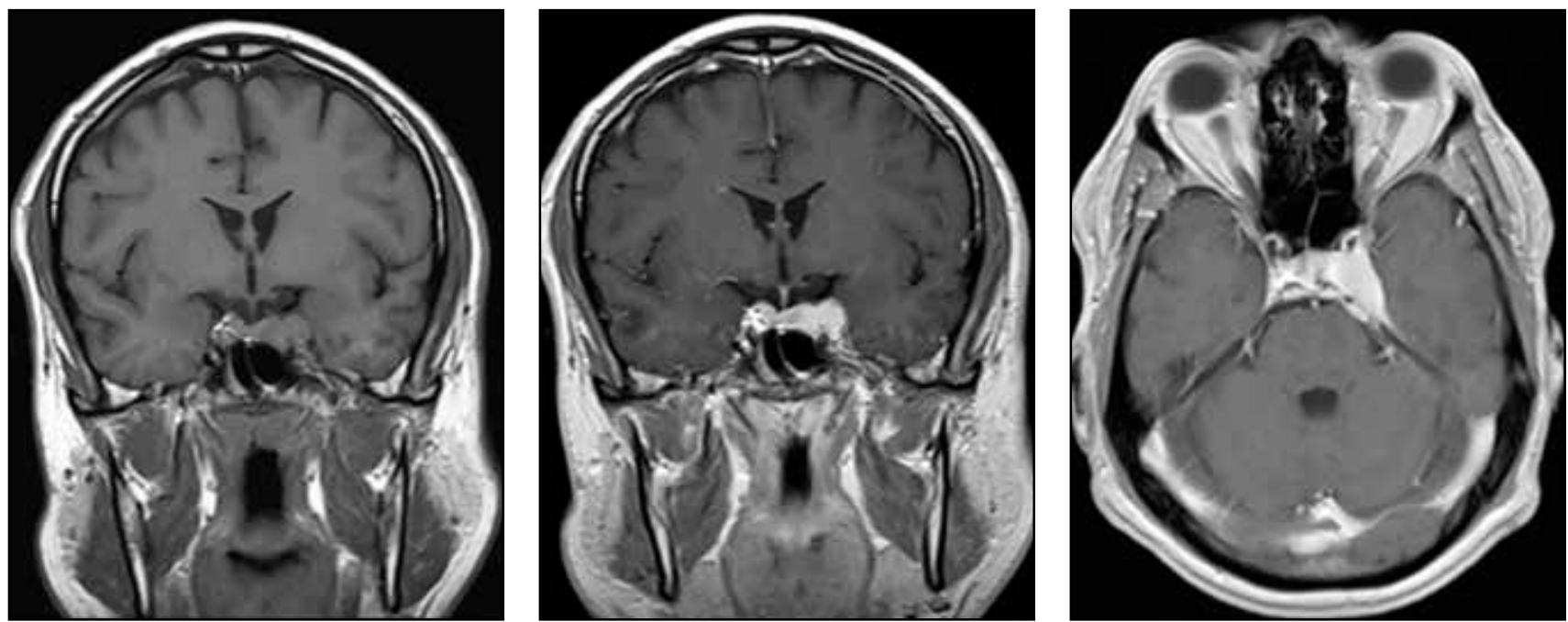

Figure $4 \mathrm{MRI}$ scan of the brain (coronal and axial section) reveals a cavernous sinus meningioma. 
extends into or infliltrates the cavernous sinus ${ }^{9,10}$.

There are still a lot of things that need to be discovered about the natural history of meningiomas. Even though they grow at a slow rate (estimated between 2 and $24 \mathrm{~mm}$ per year), there are about $23 \%$ cases of meningiomas described by various authors that did not grow (especially the calicified ones) ${ }^{11}$.

Cavernous sinus meningiomas are currently best evaluated through magnetic resonance imaging with contrast agents. These tumors are typically dural-based masses that appear to be isointense on T1-weighted and variable on T2-weighted imaging, who densely enhance and usually have a dural trail ${ }^{12}$.

Observation, microsurgical resection and stereotactic radiosurgery (gamma knife and linear accelerator) are the three main treatment options for patients with cavernous sinus meningiomas. The "watch-and-wait" strategy is based on the fact that some meningiomas can remain dormant for long periods of time and others grow only very slowly ${ }^{13-15}$. This line of treatment is usually an option for asymptomatic patients or minimally symptomatic cavernous sinus meningiomas, who exhibit cranial nerve deficit.

The primary indications for microsurgical resection are cavernous sinus meningiomas that demonstrate growth on serial imaging or become progressively more symptomatic. Stereotactic radiosurgery can also be performed in these cases as well as in asymptomatic patients. In order to avoid the risk of radiation-induced optic neuropathy, tumors compressing the optic nerves, chiasm or tracts are great candidates for resection ${ }^{16}$.

\section{CONCLUSIONS}

The particularity of this case consists in the coexistence of abducens nerve palsy with sphenoid fungal rhinosinusitis and cavernous sinus meningioma. The immediate postoperative improvement of diplopia was caused by the high doses of steroid medications during the perioperative period and not to the surgical sinus drainage.

The paper presents the limits of computed tomography versus magnetic resonance imaging in the radiologic diagnosis of intracranial tumors. Despite the fact that the patient performed not one but two CT scans, none of them identified the meningioma which has led to a delay in diagnosis. So we suggest that an MRI scan should routinely be performed in patients with neurologic pathology associated with rhinosinusitis.

With all the advances in techniques and technologies, surgeons and radio-oncologists still find a lot of challenges while managing the patients with cavernous sinus meningiomas.
Conflict of interest: The authors have no conflict of interest.

Contribution of authors: All authors have equally contributed to this work.

\section{REFERENCES}

1. Antoniades K, Karakasis D, Taskos N. Abducent nerve palsy following transverse fracture of the middle cranial fossa. J Craniomaxillo Surg. 1993;21(4):172-5.

2. Lazow SK, Izzo SR, Feinberg ME, Berger JR. Bilateral abducens nerve palsy secondary to maxillofacial trauma: report of case with proposed mechanism of injury. J Oral Maxillofac Surg. 1995;53(10):1197-9.

3. Ozveren MF, Sam B, Akdemir I, Aklan A, Tekdemir I, Deda H. Duplication of the abducens nerve at the petroclival region: an anatomic study. Neurosurgery. 2003;52(3):645-52; discussion 651-2.

4. Ziyal IM, Ozcan OE, Deniz E, Bozkurt G, Ismailoglu O. Early improvement of bilateral abducens nerve palsies following surgery of an anterior communicating artery aneurysm. Acta Neurochir (Wien). 2003;145(2):159-61; discussion 161.

5. Limnaios EE, Papageorgiou CT. Bilateral abducens nerve palsy. Ophthalmologica. 1980;181(6):326-9.

6. Nathan H, Ouaknine G, Kosary IZ. The abducens nerve. J Neurosurg. 1974;41(5):561-6.

7. Louis DN, Scheithauer BW, Budka H, von Deimling A, Kepes JJ. Meningiomas. In: Kleihues P, Cavenee WK, editors. Pathology and genetics. Tumours of the nervous system, WHO classification of tumors. Lyon: IARC Press; 2000, p.176-84.

8. Rohringer M, Sutherland GR, Louw DF, Sima AA. Incidence and clinicopathological features of meningioma. J Neurosurg. 1989;71 (5 Pt 1):66572.

9. Abdel-Aziz KM, Froelich SC, Dagnew E, Jean W, Breneman JC, et al. Large sphenoid wing meningiomas involving the cavernous sinus: conservative surgical strategies for better functional outcomes. Neurosurgery. 2004;54(6):1375-83; discussion 1383-4. DOI: 10.1227/01. NEU.0000125542.00834.6D.

10. Shrivastava RK, Sen C, Costantino PD, Della Rocca R. Sphenoorbital meningiomas: surgical limitations and lessons learned in their long-term management. I Neurosurg. 2005;103(3):491-7.

11. Oya S, Kim SH, Sade B, Lee JH. The natural history of intracranial meningiomas. J Neurosurg. 2011;114(5):1250-6. DOI: 10.3171/2010.12. JNS101623. Epub 2011 Jan 21.

12. Rokni-Yazdi H, Sotoudeh H. Prevalence of "dural tail sign" in patients with different intracranial pathologies. Eur J Radiol. 2006;60(1):42-5. Epub 2006 May 3.

13. Kuratsu J, Kochi M, Ushio Y. Incidence and clinical features of asymptomatic meningiomas. J Neurosurg. 2000;92(5):766-70.

14. Olivero WC, Lister JR, Elwood PW. The natural history and growth rate of asymptomatic meningiomas: a review of 60 patients. J Neurosurg. 1995;83(2):222-4.

15. Van Havenbergh T, Carvalho G, Tatagiba M, Plets C, Samii M. Natural history of petroclival meningiomas. Neurosurgery. 2003;52(1):55-62; discussion 62-4.

16. Pendl G, Schrottner O, Eustacchio S, Ganz JC, Feichtinger K. Cavernous sinus meningiomas - what is the strategy: upfront or adjuvant gamma knife surgery? Stereotact Funct Neurosurg. 1998;70(Suppl 1):33-40. 